\title{
Studies in humanized mice and convalescent humans yield a SARS-CoV-2 antibody cocktail
}

\begin{abstract}
Johanna Hansen ${ }^{1 *}$, Alina Baum ${ }^{1 *}$, Kristen E. Pascal'1, Vincenzo Russo ${ }^{1}$, Stephanie Giordano' Elzbieta Wloga $^{1}$, Benjamin O. Fulton', Ying Yan', Katrina Koon', Krunal Patel', Kyung Min Chung', Aynur Hermann', Erica Uliman', Jonathan Cruz ${ }^{1}$, Ashique Rafique ${ }^{1}$, Tammy Huang ${ }^{1}$, Jeanette Fairhurst ${ }^{1}$, Christen Libertiny', Marine Malbec', Wen-yi Lee', Richard Welsh', Glen Farr'1, Seth Pennington' ${ }^{1}$, Dipali Deshpande ${ }^{1}$, Jemmie Cheng'1, Anke Watty $^{1}$, Pascal Bouffard ${ }^{1}$, Robert Babb ${ }^{1}$, Natasha Levenkova ${ }^{1}$, Calvin Chen ${ }^{1}$, Bojie Zhang ${ }^{1}$, Annabel Romero Hernandez', Kei Saotome', Yi Zhou', Matthew Franklin', Sumathi Sivapalasingam', David Chien Lye ${ }^{2}$, Stuart Weston $^{3}$, James Logue ${ }^{3}$, Robert Haupt ${ }^{3}$, Matthew Frieman ${ }^{3}$, Gang Chen ${ }^{1}$, William Olson', Andrew J. Murphy', Neil Stahl ${ }^{1}$, George D. Yancopoulos ${ }^{1}$, Christos A. Kyratsous ${ }^{1} \uparrow$
\end{abstract}

${ }^{1}$ Regeneron Pharmaceuticals, Inc., Tarrytown, NY 10591, USA. ${ }^{2}$ National Centre for Infectious Diseases, Tan Tock Seng Hospital, Yong Loo Lin School of Medicine, Lee Kong Chian School of Medicine, 16 Jalan Tan Tock Seng, Singapore 308442, Singapore. 3 Department of Microbiology and Immunology, University of Maryland School of Medicine, Baltimore, MD 21201, USA.

*These authors contributed equally to this work.

†Corresponding author. Email: christos.kyratsous@regeneron.com

Neutralizing antibodies have become an important tool in treating infectious diseases. Recently, two separate approaches yielded successful antibody treatments for Ebola - one from genetically-humanized mice, and the other from a human survivor. Here, we describe parallel efforts using both humanized mice and convalescent patients to generate antibodies against the SARS-CoV-2 spike protein, yielding a large collection of fully-human antibodies that were characterized for binding, neutralization and three dimensional structure. Based on these criteria, we selected pairs of highly-potent individual antibodies that simultaneously bind the receptor-binding domain of the spike protein, providing ideal partners for a therapeutic antibody cocktail that aims to decrease the potential for virus escape mutants that might arise in response to selective pressure from a single antibody treatment.

In the setting of the current COVID19 pandemic, there has been urgency to develop potent antiviral treatments, and early efforts have hearkened back to the days of von Behring, who won the Nobel prize for showing that antibodies can be transferred in serum. However, technology advances over the last century have allowed for the progression from convalescent serum to the utilization of recombinant fully human antibodies. The proposal to genetically humanize the immune system of mice (1) provided an efficient source of naturallyselected fully-human antibodies. For example, such mice were used to develop checkpoint inhibitors for immune- oncology (2), as well as FDA-approved antibodies for the treatment of rheumatoid arthritis, cardiovascular disease, cutaneous squamous cell carcinoma, and allergic diseases such as asthma and atopic dermatitis. More recently, the ability to sort individual B cells from previously infected human patients - and molecularly clone the antibody genes from these B cells - has led to an independent source of human antibodies, albeit limited to antibodies targeting infectious agents. Recently, these two fundamentally different approaches were independently exploited to develop fully-human antibody treatments for the lethal infectious disease caused by the Ebola virus: genetically humanized "VelocImmune ${ }^{\circledR}$ " (VI) mice $(3,4)$ generated an Ebola antibody cocktail treatment (5), while sorting of B cells from a recovered patient yielded a single therapeutic antibody treatment (6).

Here we describe parallel high-throughput efforts using both mice and humans to generate antibodies against the spike protein of SARS-CoV-2. The ability to derive antibodies using genetically humanized VI mice as well as B cells derived from convalescent patients enabled us to isolate a very large collection of fully human antibodies with diverse sequences, binding properties, and antiviral activity. The prospective goal of these parallel efforts was to generate a large and diverse collection so as to allow for the selection of pairs of highly potent individual antibodies that could simultaneously bind the critical Receptor Binding Domain (RBD) of the spike protein, providing ideal partners for a therapeutic antibody cocktail that would have the potential to decrease the likelihood of virus escape mutants that might arise in response to selective pressure from single antibody treatments (7).

Anti-SARS-CoV-2 spike antibodies were generated with the following two methods. First, by immunizing VI mice 
with a DNA plasmid that expresses SARS-CoV-2 spike protein and then boosting with recombinant protein comprised of the RBD of SARS-CoV-2 spike. Second, by isolation from PBMCs of human donors previously infected with SARS-CoV2. VI mice elicited a robust immune response against the SARS-CoV-2 spike protein post-immunization. Titers of the bleeds of the mice collected 7 days after the last boost were determined by ELISA (fig. S1). Mice with the highest titers were used for antibody isolation. Spleens from these mice were subjected to biotin-labeled monomeric RBD antigen staining, and FACS single cell sorting. In parallel, whole blood was received from 3 patients 3-4 weeks post laboratoryconfirmed PCR positive test for SARS-CoV-2 and symptomatic COVID-19 disease; PBMCs were isolated by ficoll gradient and RBD-specific B cells were FACS single cell sorted. The first sets of antibodies derived from these platforms are described herein.

To assess antigen specific responses, naturally-paired heavy and light chain cDNAs were cloned from the mouse and human derived B cells (8) and transfected into Chinese hamster ovary (CHO) cells to produce recombinant fully-human antibodies. Cultured supernatants containing secreted antibodies were subjected to high-throughput screening for $\mathrm{RBD}$ protein binding. Thousands of antibodies were isolated and subsequently screened for binding affinity to RBD monomer and dimer, epitope diversity, ability to block ACE2 receptor binding to RBD, and neutralize VSV-based SARS-CoV2 spike pseudoparticles (pVSV-SARS-CoV-2-S(mNeon)). Screening yielded over 200 neutralizing mAbs with broad potency ranges, dozens of which displayed neutralization potency in the $\mathrm{pM}$ range.

Over 200 of the VI and human derived antibodies isolated in the primary screen neutralized VSV-based SARS-CoV-2 Spike pseudoparticles at $>70 \%$ with $\sim 2 \mu \mathrm{g} / \mathrm{ml}$ of expressed antibodies. The antibody variable regions were sequenced by Next Generation Sequencing and the repertoire for heavy and light chain pairs was identified (Fig. 1). The predominant lineage of VI antibodies utilized VH3-53 paired with VK1-9, VK133 , or VK1-39, while our human-derived antibodies utilized VH3-66 paired with VK1-33 or VH2-70 paired with VK1-39. Interestingly, VH3-53 usage has recently been reported for another human-derived potent neutralizing antibody against SARS-CoV-2 spike protein (9-11), indicating that combining the VI mouse approach with the human platforms allows expanded capture of common rearrangements found in potent neutralizing SARS-CoV-2 mAbs seen in humans. Further analysis of overlaid sequences (fig. S2.) showed strong overlap in the repertoire of isolated kappa chains between VI and human-derived antibodies. Although the repertoire of Lambda chains did not overlap well that may be due to only two lambda mice being included in this trial. The average CDR length (fig. S2D) for heavy chain was similar between VI and human derived antibodies with an average length of 13 and 14.5 amino acids, respectively. Average kappa CDR length (fig. S2E) was the same for VI and human derived antibodies at 9 amino acids and was close for lambda chains (fig. S2F) with an average length of 11.1 and 10.6 amino acids, respectively.

Approximately 40 antibodies with unique sequences and potent neutralization activity were chosen for additional characterization as described below. The neutralization potency of these mAbs spanned the single digit to triple digit $\mathrm{pM}$ range in the VSV-based pseudoparticle assay. While we did identify antibodies that bound to the SARS-CoV-1 spike protein (data not shown), these were weakly neutralizing, supporting previously published reports describing SARSCoV-1 and SARS-CoV-2 cross-neutralizers (12). Instead we further focused on nine of the most potent neutralizing mAbs, with neutralization potencies ranging from $7 \mathrm{pM}$ to 99 pM (Fig. 2A and table S1). All of these neutralizing mAbs bound to the RBD of SARS-CoV-2 spike and blocked its ability to interact with ACE2 with double-digit pM IC50s (table S1), supporting ACE2 blockade as the primary mechanism for neutralization. The antibodies bound specifically and with high affinity to monomeric SARS-COV-2 RBD (KD $=0.56$ to $45.2 \mathrm{nM})$ and dimeric SARS-COV-2 $\mathrm{RBD}(\mathrm{KD}=5.7$ to $42.8 \mathrm{pM})$. As recombinant ACE2 receptor is being considered as a COVID19 therapeutic (13), we tested the potency of recombinant dimeric human ACE2-Fc (hACE2-hFc) in our neutralization assay. While recombinant ACE2 was able to mediate neutralization of the VSV-spike pseudoparticles as previously reported, it was more than 1000 -fold less potent than the best neutralizing mAbs (Fig. 2, A and B).

A smaller collection of four antibodies was chosen for further analyses to determine whether the above binding data to $\mathrm{RBD}$ reflected binding to trimeric spike protein, whether neutralization potencies noted in the above assays were consistent with those seen in other assays including with SARSCoV-2, and whether these antibodies retained neutralization activity against pseudoparticles with mutations in the S1/S2 cleavage site. Binding affinity of these four antibodies against trimeric SARS-CoV-2 spike ( $\mathrm{KD}=37.1$ to $42.8 \mathrm{pM})$ largely paralleled the affinity against the RBD (table S3). In addition, the potent neutralizing activity of these four antibodies was confirmed in the additional neutralization assays, including pVSV-SARS-CoV-2-S(mNeon) neutralization in the human lung epithelial Calu3 cell line, neutralization of replicating VSV-SARS-CoV-2-S in Vero cells, and neutralization of SARSCoV-2 in VeroE6 cells (Fig. 2, B to D). All neutralization assays generated similar potency across the four mAbs and no combinations demonstrated synergistic neutralization activity (Fig. 2, C and D). As previous reports indicate pseudoparticles containing the SARS-CoV-2 Spike are pre-cleaved by furinlike proteases at the polybasic S1/S2 cleavage site during 
biogenesis in HEK293T cells, we assessed the impact of this cleavage on mAb neutralization potency. Spike stabilized pseudoparticles (fig. S3A) with a monobasic cleavage site (FurMut) in the S1/S2 interface or deleted region (FurKO) were produced as previously described $(14,15)$. No differences were observed in neutralization of either FurMut or FurKO containing pseudoparticles relative to wild-type (WT) in Vero cells (fig. S3B). Interestingly, stabilized pseudoparticles had comparable or greater infectivity to those with WT cleavage sites in Vero cells, whereas dramatic loss of infectivity was observed in Calu-3 cells (fig. S3C). Authentic SARS-CoV-2 with a natural deletion of the S1/S2 junction also had defects in infectivity in Calu-3, but not in Vero cells (16), implicating differential protease usage between these two cell types. To investigate the mechanism of neutralization we generated Fab fragments for the four antibodies. We compared IgG to corresponding Fabs side-by-side for their ability to neutralize pseudotyped VSV (fig. S4). The IC50s of all the Fabs was shifted compared to their parental IgG, indicating that bivalent binding, cross-linking and steric hindrance might all augment neutralization.

Although the role of antibody effector function in protection against SARS-CoV-2 is yet unknown, it is well established to play an important role in $\mathrm{mAb}$ therapeutic efficacy against other viruses such as Ebola and influenza viruses (17-19). Effector cells including macrophages and monocytes were also shown to be important for antibody mediated protection from SARS-CoV-1 infection (20). To understand whether our lead antibodies are capable of mediating effector function, we assessed both Antibody Dependent Cellular Cytotoxicity (ADCC) and Antibody Dependent Cellular Phagocytosis (ADCP) activity in primary human cell bioassays utilizing NK cells and monocyte derived phagocytes. All four lead antibodies demonstrated the ability to mediate ADCC and ADCP, albeit to slightly different degrees. REGN10987 displayed superior ability to mediate $\mathrm{ADCC}$ relative to the other three mAbs, while it performed similarly to REGN10989 and REGN10933 in the ADCP assay (fig. S5 and table S3). Although REGN10934 was able to mediate both ADCC and $\mathrm{ADCP}$, it was not as strong of an inducer in those assays as the other three mAbs (fig. S6 and table S4). Further identification of $\mathrm{mAb}$ epitopes through high resolution structural analysis may help illuminate the relationship between specific epitopes and effector function of anti-spike mAbs.

A prospective goal of our effort was to identify highly potent individual antibodies that could be paired in a therapeutic antibody cocktail, aiming to decrease the potential for decreased efficacy due to variants arising as the pandemic spreads, as well as due to virus escape mutants that might be selected in response to pressure from a single antibody treatment (7). We thus examined our nine most potent neutralizing antibodies in cross-competition binding assays (fig. S7), identifying several pairs of non-competing mAbs with pM neutralization potency that could potentially be combined to form antibody cocktails. To further study the binding regions of our mAbs on spike protein RBD, we performed HydrogenDeuterium Exchange Mass Spectrometry (HDX-MS) with the same nine antibodies (Fig. 3), revealing where each of the antibodies contacts the surface of the RBD, and allowing comparison with the ACE2 binding site on the RBD (Fig. 3). As might be expected, most of our neutralizing antibodies contact the RBD in a manner that overlaps the RBD residues that comprise the ACE2 interface; furthermore, the antibodies can be grouped based on their pattern of contacting the RBD surface. Comparing the cross-competition binding assays with the HDX-MS results provides structural insights into the mechanism by which non-competing pairs of antibodies can simultaneously bind the RBD, and can thus be ideal partners for a therapeutic antibody cocktail. REGN10987 and REGN10933 represent such a pair of antibodies: REGN10933 targets the spike-like loop region on one edge of the ACE2 interface. Within that region, the residues that show the most significant HDX protection by REGN10933 face upward, suggesting that the Fab region of REGN10933 binds the RBD from the top direction, where REGN10933 will have significant collisions with ACE2. In order to avoid competition with REGN10933, REGN10987 can only bind to the HDX defined protected regions from the front or the lower left side (in the front view of REGN10987 in Fig. 3). This would be consistent with the neutralization data, as REGN10987 would orient itself in a position that has high probability to interfere with ACE2. Confirming the above data, single-particle cryoEM of the complex of SARS-CoV-2 spike RBD bound to Fab fragments of REGN10933 and REGN10987 shows that the two antibodies in this cocktail can simultaneously bind to distinct regions of the RBD (Fig. 4 and table S5). A 3D reconstructed map of the complex with nominal resolution of $3.9 \AA$ shows that the two Fab fragments bind at different epitopes on the RBD confirming that they are non-competing antibodies. REGN10933 binds at the top of the RBD, extensively overlapping the binding site for ACE2. On the other hand, the epitope for REGN10987 is located on the side of the RBD, away from the REGN10933 epitope, and has little to no overlap with the ACE2 binding site.

We report remarkable similarities and consistencies in the antibodies generated from genetically-humanized mice and from convalescent humans. The scale of the genetic-engineering approach used to create the VI mouse (involving genetic-humanization of over 6 mega-bases of mouse immune genes) has resulted in the ability to effectively and indistinguishably mimic the antibody responses of normal humans. The genetically-humanized mouse approach has the advantage that it can potentially allow for further immunization optimization strategies, and also that it can be applied 
to non-infectious disease targets. By combining the efforts from two parallel and high-throughput approaches for generating antibodies to the RBD of the SARS-CoV-2 spike protein, we generated a sufficiently large collection of potent and diverse antibodies that we could meet our prospective goal of identifying highly potent individual antibodies that could be combined into a therapeutic antibody cocktail. Inclusion of such antibodies into an antibody cocktail may deliver optimal antiviral potency while minimizing odds of virus escape (7), two critical desired features of an antibody-based therapeutic for treatment and prevention of COVID19. Such an antibody cocktail is now being tested in human trials (clinicaltrials.gov NCT04426695 and NCT04425629).

\section{REFERENCES AND NOTES}

1. F. W. Alt, T. K. Blackwell, G. D. Yancopoulos, Immunoglobulin genes in transgenic mice. Trends Genet. 1, 231-236 (1985). doi:10.1016/0168-9525(85)90089-7

2. J. Larkin, V. Chiarion-Sileni, R. Gonzalez, J. J. Grob, C. L. Cowey, C. D. Lao, D. Schadendorf, R. Dummer, M. Smylie, P. Rutkowski, P. F. Ferrucci, A. Hill, J. Wagstaff, M. S. Carlino, J. B. Haanen, M. Maio, I. Marquez-Rodas, G. A. McArthur, P. A. Ascierto, G. V. Long, M. K. Callahan, M. A. Postow, K. Grossmann, M. Sznol, B. Dreno, L. Bastholt, A. Yang, L. M. Rollin, C. Horak, F. S. Hodi, J. D. Wolchok, Combined Nivolumab and Ipilimumab or Monotherapy in Untreated Melanoma. N. Engl. J. Med. 373, 23-34 (2015). doi:10.1056/NEJMoa1504030 Medline

3. A. J. Murphy, L. E. Macdonald, S. Stevens, M. Karow, A. T. Dore, K. Pobursky, T. T. Huang, W. T. Poueymirou, L. Esau, M. Meola, W. Mikulka, P. Krueger, J. Fairhurst, D. M. Valenzuela, N. Papadopoulos, G. D. Yancopoulos, Mice with megabase humanization of their immunoglobulin genes generate antibodies as efficiently as normal mice. Proc. Natl. Acad. Sci. U.S.A. 111, 5153-5158 (2014). doi:10.1073/pnas.1324022111 Medline

4. L. E. Macdonald, M. Karow, S. Stevens, W. Auerbach, W. T. Poueymirou, J. Yasenchak, D. Frendewey, D. M. Valenzuela, C. C. Giallourakis, F. W. Alt, G. D. Yancopoulos, A. J. Murphy, Precise and in situ genetic humanization of $6 \mathrm{Mb}$ of mouse immunoglobulin genes. Proc. Natl. Acad. Sci. U.S.A. 111, 5147-5152(2014). doi:10.1073/pnas.1323896111 Medline

5. K. E. Pascal, D. Dudgeon, J. C. Trefry, M. Anantpadma, Y. Sakurai, C. D. Murin, H. L. Turner, J. Fairhurst, M. Torres, A. Rafique, Y. Yan, A. Badithe, K. Yu, T. Potocky, S. L. Bixler, T. B. Chance, W. D. Pratt, F. D. Rossi, J. D. Shamblin, S. E. Wollen, J. M. Zelko, R. Carrion Jr., G. Worwa, H. M. Staples, D. Burakov, R. Babb, G. Chen, J. Martin, T. T. Huang, K. Erlandson, M. S. Willis, K. Armstrong, T. M. Dreier, A. B. Ward, R. A. Davey, M. L. M. Pitt, L. Lipsich, P. Mason, W. Olson, N. Stahl, C. A. Kyratsous, Development of Clinical-Stage Human Monoclonal Antibodies That Treat Advanced Ebola Virus Disease in Nonhuman Primates. J. Infect. Dis. 218, S612-S626 (2018). doj:10.1093/infdis/jiy285 Medline

6. D. Corti, J. Misasi, S. Mulangu, D. A. Stanley, M. Kanekiyo, S. Wollen, A. Ploquin, N. A. Doria-Rose, R. P. Staupe, M. Bailey, W. Shi, M. Choe, H. Marcus, E. A. Thompson, A. Cagigi, C. Silacci, B. Fernandez-Rodriguez, L. Perez, F. Sallusto, F. Vanzetta, G. Agatic, E. Cameroni, N. Kisalu, I. Gordon, J. E. Ledgerwood, J. R. Mascola, B. S. Graham, J.-J. Muyembe-Tamfun, J. C. Trefry, A. Lanzavecchia, N. J. Sullivan, Protective monotherapy against lethal Ebola virus infection by a potently neutralizing antibody. Science 351, 1339-1342 (2016). doi:10.1126/science.aad5224 Medline

7. A. Baum, B. O. Fulton, E. Wloga, R. Copin, K. E. Pascal, V. Russo, S. Giordano, K. Lanza, N. Negron, M. Ni, Y. Wei, G. S. Atwal, A. J. Murphy, N. Stahl, G. D. Yancopoulos, C. A. Kyratsous, Antibody Cocktail to SARS-Cov-2 Spike Protein Prevents Rapid Mutational Escape Seen with Individual Antibodies, Science 10.1126/science.abd0831 (2020).

8. X. Wang, B. D. Stollar, Human immunoglobulin variable region gene analysis by single cell RT-PCR. J. Immunol. Methods 244, 217-225 (2000). doi:10.1016/S0022-1759(00)00260-X Medline

9. Y. Cao, B. Su, X. Guo, W. Sun, Y. Deng, L. Bao, Q. Zhu, X. Zhang, Y. Zheng, C. Geng, X. Chai, R. He, X. Li, Q. Lv, H. Zhu, W. Deng, Y. Xu, Y. Wang, L. Qiao, Y. Tan, L. Song,
G. Wang, X. Du, N. Gao, J. Liu, J. Xiao, X. D. Su, Z. Du, Y. Feng, C. Qin, C. Qin, R. Jin, $X$. S. Xie, Potent neutralizing antibodies against SARS-CoV-2 identified by highthroughput single-cell sequencing of convalescent patients' B cells. Cell 10.1016/j.cell.2020.05.025 (2020). doi:10.1016/j.cell.2020.05.025 Medline

10. Y. Wu, F. Wang, C. Shen, W. Peng, D. Li, C. Zhao, Z. Li, S. Li, Y. Bi, Y. Yang, Y. Gong, H. Xiao, Z. Fan, S. Tan, G. Wu, W. Tan, X. Lu, C. Fan, Q. Wang, Y. Liu, C. Zhang, J. Qi, G. F. Gao, F. Gao, L. Liu, A noncompeting pair of human neutralizing antibodies block COVID-19 virus binding to its receptor ACE2. Science 368, 1274-1278 (2020). doi:10.1126/science.abc2241 Medline

11. T. F. Rogers, F. Zhao, D. Huang, N. Beutler, A. Burns, W.-T. He, O. Limbo, C. Smith, G. Song, J. Woehl, L. Yang, R. K. Abbott, S. Callaghan, E. Garcia, J. Hurtado, M. Parren, L. Peng, S. Ramirez, J. Ricketts, M. J. Ricciardi, S. A. Rawlings, N. C. Wu, M. Yuan, D. M. Smith, D. Nemazee, J. R. Teijaro, J. E. Voss, I. A. Wilson, R. Andrabi, B. Briney, E. Landais, D. Sok, J. G. Jardine, D. R. Burton, Isolation of potent SARSCoV-2 neutralizing antibodies and protection from disease in a small animal model, Science 10.1126/science.abc7520 (2020).

12. M. Yuan, N. C. Wu, X. Zhu, C. D. Lee, R. T. Y. So, H. Lv, C. K. P. Mok, I. A. Wilson, A highly conserved cryptic epitope in the receptor binding domains of SARS-CoV-2 and SARS-CoV. Science 368, 630-633 (2020). doi:10.1126/science.abb7269 Medline

13. C. Lei, K. Qian, T. Li, S. Zhang, W. Fu, M. Ding, S. Hu, Neutralization of SARS-CoV-2 spike pseudotyped virus by recombinant ACE2-Ig. Nat. Commun. 11, 2070 (2020). doi:10.1038/s41467-020-16048-4 Medline

14. A. C. Walls, Y.-J. Park, M. A. Tortorici, A. Wall, A. T. McGuire, D. Veesler, Structure, Function, and Antigenicity of the SARS-CoV-2 Spike Glycoprotein. Cell 181, 281292.e6 (2020). doi:10.1016/j.cell.2020.02.058

15. D. Wrapp, N. Wang, K. S. Corbett, J. A. Goldsmith, C.-L. Hsieh, O. Abiona, B. S. Graham, J. S. McLellan, Cryo-EM structure of the 2019-nCoV spike in the prefusion conformation. Science 367, 1260-1263 (2020). doi:10.1126/science.abb2507 Medline

16. S. Y. Lau, P. Wang, B. W.-Y. Mok, A. J. Zhang, H. Chu, A. C.-Y. Lee, S. Deng, P. Chen, K.-H. Chan, W. Song, Z. Chen, K. K.-W. To, J. F.-W. Chan, K.-Y. Yuen, H. Chen, Attenuated SARS-CoV-2 variants with deletions at the S1/S2 junction. Emerg. Microbes Infect. 9, 837-842 (2020). doi:10.1080/22221751.2020.1756700 Medline

17. D. J. DiLillo, P. Palese, P. C. Wilson, J. V. Ravetch, Broadly neutralizing antiinfluenza antibodies require $F c$ receptor engagement for in vivo protection. J. Clin. Invest. 126, 605-610 (2016). doj:10.1172/JCl84428 Medline

18. E. O. Saphire, S. L. Schendel, B. M. Gunn, J. C. Milligan, G. Alter, Antibody-mediated protection against Ebola virus. Nat. Immunol. 19, 1169-1178 (2018). doi:10.1038/s41590-018-0233-9 Medline

19. E. O. Saphire, S. L. Schendel, M. L. Fusco, K. Gangavarapu, B. M. Gunn, A. Z. Wec, P. J. Halfmann, J. M. Brannan, A. S. Herbert, X. Qiu, K. Wagh, S. He, E. E. Giorgi, J. Theiler, K. B. J. Pommert, T. B. Krause, H. L. Turner, C. D. Murin, J. Pallesen, E. Davidson, R. Ahmed, M. J. Aman, A. Bukreyev, D. R. Burton, J. E. Crowe Jr., C. W. Davis, G. Georgiou, F. Krammer, C. A. Kyratsous, J. R. Lai, C. Nykiforuk, M. H. Pauly, P. Rijal, A. Takada, A. R. Townsend, V. Volchkov, L. M. Walker, C.-I. Wang, L. Zeitlin, B. J. Doranz, A. B. Ward, B. Korber, G. P. Kobinger, K. G. Andersen, Y. Kawaoka, G. Alter, K. Chandran, J. M. Dye, Viral Hemorrhagic Fever Immunotherapeutic Consortium, Systematic Analysis of Monoclonal Antibodies against Ebola Virus GP Defines Features that Contribute to Protection. Cell 174, 938-952.e13 (2018). doi:10.1016/j.cell.2018.07.033 Medline

20. F. Yasui, M. Kohara, M. Kitabatake, T. Nishiwaki, H. Fujii, C. Tateno, M. Yoneda, K. Morita, K. Matsushima, S. Koyasu, C. Kai, Phagocytic cells contribute to the antibody-mediated elimination of pulmonary-infected SARS coronavirus. Virology 454-455, 157-168 (2014). doi:10.1016/j.virol.2014.02.005 Medline

21. R. Yan, Y. Zhang, Y. Li, L. Xia, Y. Guo, Q. Zhou, Structural basis for the recognition of SARS-CoV-2 by full-length human ACE2. Science 367, 1444-1448 (2020). doi:10.1126/science. abb2762 Medline

22. J. Lan, J. Ge, J. Yu, S. Shan, H. Zhou, S. Fan, Q. Zhang, X. Shi, Q. Wang, L. Zhang, X. Wang, Structure of the SARS-CoV-2 spike receptor-binding domain bound to the ACE2 receptor. Nature 581, 215-220 (2020). doj:10.1038/s41586-020-2180-5 Medline

23. N. C. Shaner, G. G. Lambert, A. Chammas, Y. Ni, P. J. Cranfill, M. A. Baird, B. R. Sell, J. R. Allen, R. N. Day, M. Israelsson, M. W. Davidson, J. Wang, A bright monomeric 
green fluorescent protein derived from Branchiostoma lanceolatum. Nat. Methods 10, 407-409 (2013). doi:10.1038/nmeth.2413 Medline

24. N. D. Lawson, E. A. Stillman, M. A. Whitt, J. K. Rose, Recombinant vesicular stomatitis viruses from DNA. Proc. Natl. Acad. Sci. U.S.A. 92, 4477-4481 (1995). doi:10.1073/pnas.92.10.4477 Medline

25. E. A. Stillman, J. K. Rose, M. A. Whitt, Replication and amplification of novel vesicular stomatitis virus minigenomes encoding viral structural proteins. J Virol. 69, 2946-2953 (1995). Medline

26. M. A. Whitt, Generation of VSV pseudotypes using recombinant $\Delta G-V S V$ for studies on virus entry, identification of entry inhibitors, and immune responses to vaccines. J. Virol. Methods 169, 365-374 (2010). doi:10.1016/ijviromet 2010.08.006 Medline

27. A. Takada, C. Robison, H. Goto, A. Sanchez, K. G. Murti, M. A. Whitt, Y. Kawaoka, A system for functional analysis of Ebola virus glycoprotein. Proc. Natl. Acad. Sci. U.S.A. 94, 14764-14769 (1997). doi:10.1073/pnas.94.26.14764 Medline

28. S. Fukushi, T. Mizutani, M. Saijo, S. Matsuyama, N. Miyajima, F. Taguchi, S. Itamura, I. Kurane, S. Morikawa, Vesicular stomatitis virus pseudotyped with severe acute respiratory syndrome coronavirus spike protein. J. Gen. Virol. 86, 2269-2274 (2005). doi:10.1099/vir.0.80955-0 Medline

29. J. Nie, Q. Li, J. Wu, C. Zhao, H. Hao, H. Liu, L. Zhang, L. Nie, H. Qin, M. Wang, Q. Lu, X. Li, Q. Sun, J. Liu, C. Fan, W. Huang, M. Xu, Y. Wang, Establishment and validation of a pseudovirus neutralization assay for SARS-CoV-2. Emerg. Microbes Infect. 9, 680-686 (2020). doi:10.1080/22221751.2020.1743767 Medline

30. B. Johnsson, S. Löfås, G. Lindquist, Immobilization of proteins to a carboxymethyldextran-modified gold surface for biospecific interaction analysis in surface plasmon resonance sensors. Anal. Biochem. 198, 268-277 (1991). doi:10.1016/0003-2697(91)90424-R Medline

31. D. G. Myszka, Improving biosensor analysis. J. Mol. Recognit. 12, 279-284 (1999). doi:10.1002/(SICI)1099-1352(199909/10)12:5<279:AID-JMR473>3.0.CO:2-3 Medline

32. Y. N. Abdiche, D. S. Malashock, A. Pinkerton, J. Pons, Exploring blocking assays using Octet, ProteOn, and Biacore biosensors. Anal. Biochem. 386, 172-180 (2009). doi:10.1016/j.ab.2008.11.038 Medline

33. A. Punjani, J. L. Rubinstein, D. J. Fleet, M. A. Brubaker, cryoSPARC: Algorithms for rapid unsupervised cryo-EM structure determination. Nat. Methods 14, 290-296 (2017). doi:10.1038/nmeth.4169 Medline

\section{ACKNOWLEDGMENTS}

Authors would like to thank Kristen Tramaglini for program management. Funding: A portion of this project has been funded in whole or in part with Federal funds from the Department of Health and Human Services; Office of the Assistant Secretary for Preparedness and Response; Biomedical Advanced Research and Development Authority, under OT number: HHSO100201700020C. Author contributions: J.H., A.B., B.O.F, A.H., E.U., A.R., T.H., J.F., P.B., R.B., B.Z., M.F., A.J.M., N.S, G.D.Y., C.A.K conceptualized and designed experiments. J.H., K.E.P., V.R., S.G., E.W., B.O.F., Y.Y., K.K., K.P., K.M.C., E.U., J.C., A.R., C.L., M.M., W.L., R.W., S.P., J.C., B.Z., A.R.H., K.S., Y.Z., M.F., S.W., J.L.,R.H.,M.F. performed research and J.H., K.E.P., V.R., S.G., E.W., B.O.F., Y.Y., K.K., K.P., K.M.C., E.U., J.C., A.R., J.F., C.L., M.M., W.L., G.F., D.D., J.C., P.B., R.B., N.L., C.C., B.Z., A.R.H., K.S., Y.Z., M.F., S.W., J.L., R.H., M.F., G.C., W.O., N.S., G.D.Y., C.A.K. analyzed data. S.S, D.C.L. acquired reagents . J.H., A.B., A.R., B.Z., M.F., G.D.Y., C.A.K. wrote the paper. C.A.K. acquired funding. Competing interests: Regeneron authors own options and/or stock of the company. This work has been described in one or more pending provisional patent applications. GC, WO, AJM, NS, GDY and CAK are officers of Regeneron. Data and materials availability: The structure of SARS-CoV-2 RBD in complex with REGN10933 and REGN10987 Fabs has been deposited in the Protein Data Bank, with PDB ID 6XDG. The corresponding cryoEM map is available in the Electron Microscopy Data Bank, with ID EMD-22137. Sequences of the nine characterized antibodies have been deposited in GenBank and are available in the supplementary materials. Regeneron materials described in this manuscript may be made available to qualified, academic, noncommercial researchers through a material transfer agreement upon request at https://regeneron.envisionpharma.com/vt_regeneron/. For questions about how Regeneron shares materials, use the email address (preclinical.collaborations@ regeneron.com).This work is licensed under a Creative Commons Attribution 4.0 International (CC BY 4.0) license, which permits unrestricted use, distribution, and reproduction in any medium, provided the original work is properly cited. To view a copy of this license, visit https:/creativecommons.org/licenses/by/4.0/. This license does not apply to figures/photos/artwork or other content included in the article that is credited to a third party; obtain authorization from the rights holder before using such material.

\section{SUPPLEMENTARY MATERIALS}

science.sciencemag.org/cgi/content/full/science.abd0827/DC1

Materials and Methods

Figs. S1 to S7

Tables S1 to S5

References (23-33)

MDAR Reproducibility Checklist

Data S1

30 May 2020; accepted 11 June 2020

Published online 15 June 2020

10.1126/science.abd0827 
A

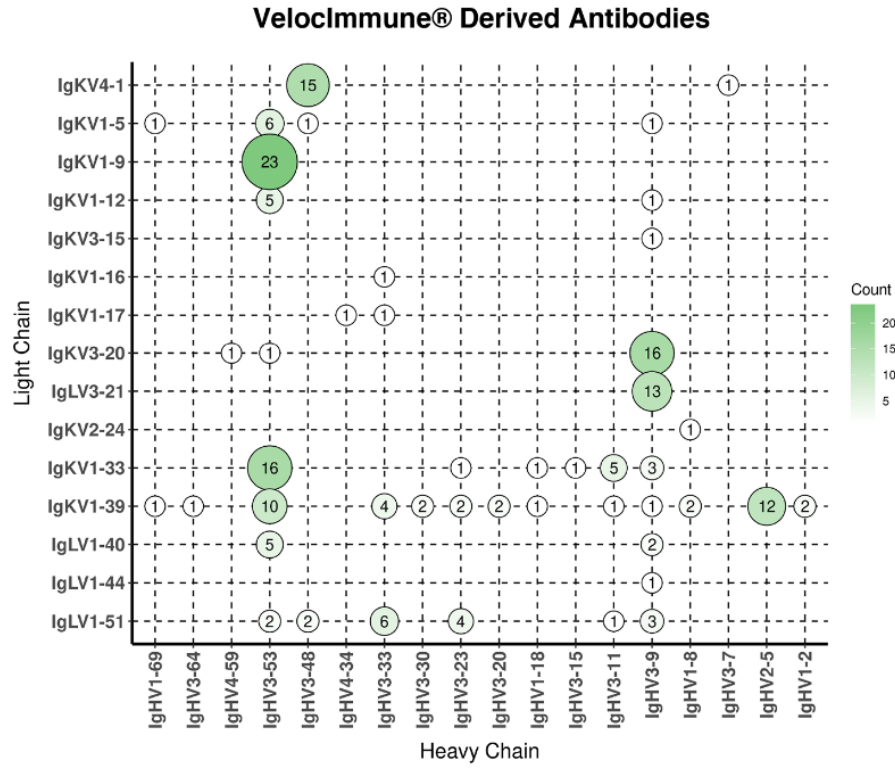

B

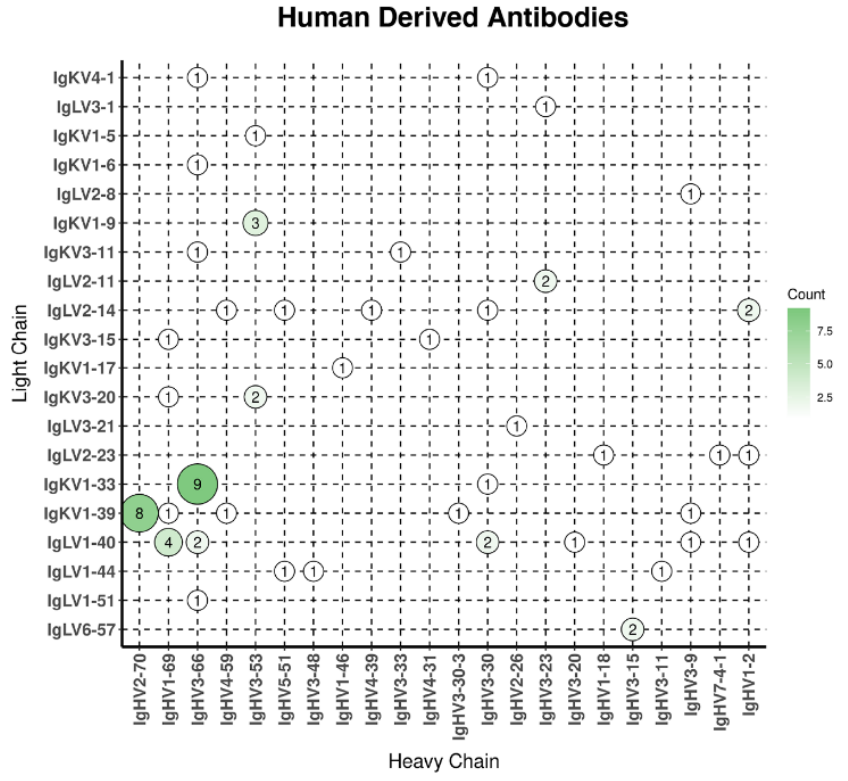

Fig. 1. Paired antibody repertoire for human and mouse-derived SARS-CoV-2 neutralizing antibodies. (A and $B) \vee$ gene frequencies for paired Heavy ( $X$-axis) and Light ( $Y$-axis) chains of isolated neutralizing antibodies to SARS-CoV-2 for Veloclmmune ${ }^{\circledR}$ mice (Left panel; $\mathrm{N}=185$ ) and convalescent human donors (Right panel; $\mathrm{N}=68$ ). The color and size of the circle corresponds to the number of Heavy and Light chain pairs present in the repertoires of isolated neutralizing antibodies. Neutralization is defined as $>70 \%$ with $1: 4$ dilution of antibody $(\sim 2 \mathrm{ug} / \mathrm{ml})$ in VSV pseudoparticle neutralization assay. 
A

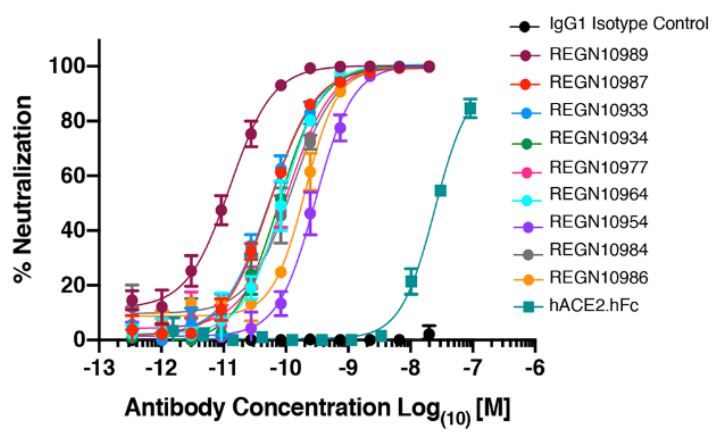

C

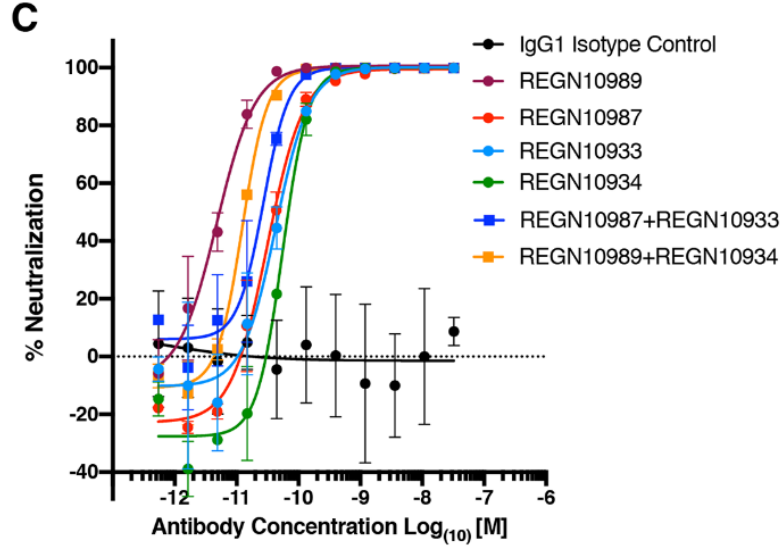

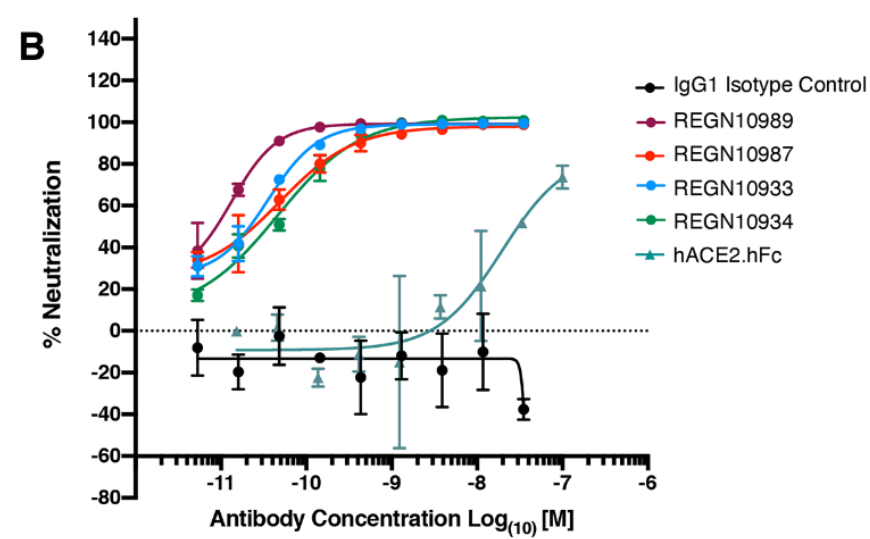

D

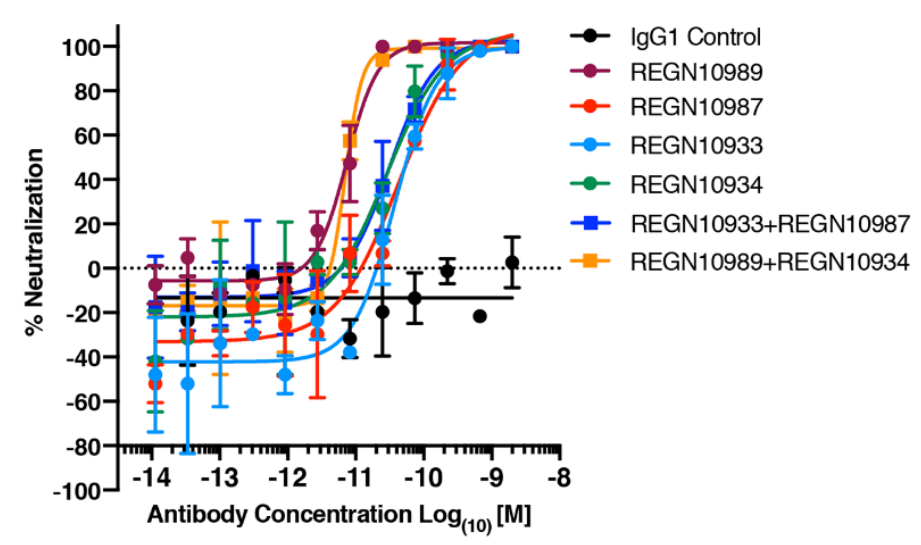

Fig. 2. Neutralization potency of anti-SARS-CoV-2 spike mAbs. (A) Serial dilutions of anti-spike mAbs, IgG1 isotype control, and recombinant dimeric ACE2 (hACE2.hFc) were added with pVSV-SARS-CoV-2-S-mNeon to Vero cells and mNeon expression was measured 24 hours post-infection as a read-out for virus infectivity. Data are graphed as percent neutralization relative to virus only infection control. (B) Neutralization potency of antispike mAbs, recombinant dimeric ACE2, and IgG1 isotype control against non-replicating pVSV-SARS-CoV-2-SmNeon in Calu3 cells. (C) Neutralization potency of individual anti-spike mAbs and combinations of mAbs against replicating VSV-SARS-CoV-2-S virus in Vero cells. Cells were infected with an MOI 1 of the virus and stained for viral protein 24 hours post-infection to measure infectivity. (D) Neutralization potency of individual anti-spike mAbs and combinations of mAbs against SARS-CoV-2-S virus in VeroE6 cells. 

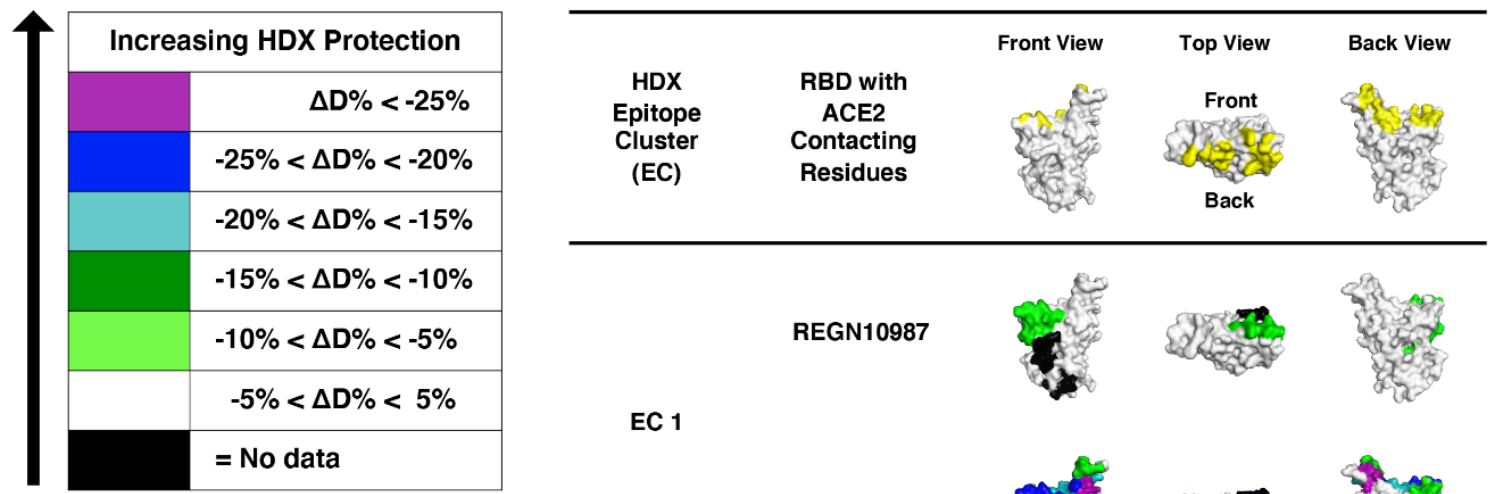

EC 1

RC 2

Fig. 3. Hydrogen-Deuterium Exchange Mass Spectrometry determines mAb interaction on Spike protein RBD. 3D surface model for the structure of the Spike protein RBD domain showing the ACE2 interface and HDX-MS epitope mapping results. RBD residues that make contacts with ACE2 $(21,22)$ are indicated in yellow (top panel). RBD residues protected by antiSARS-CoV2-Spike antibodies are indicated with colors that represent the extent of protection as determined by HDX-MS experiments. RBD residues in purple and blue indicate sites of lessor solvent exchange upon antibody binding and have greater likelihood to be antibody binding residues. The RBD structure is reproduced from PDB 6M17 (21). 
A

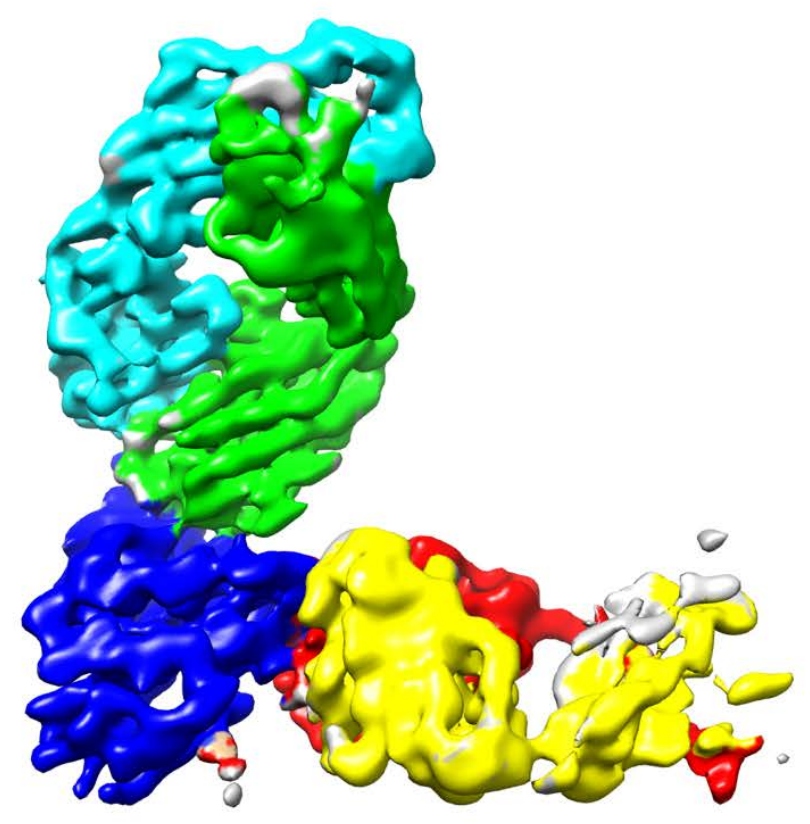

B

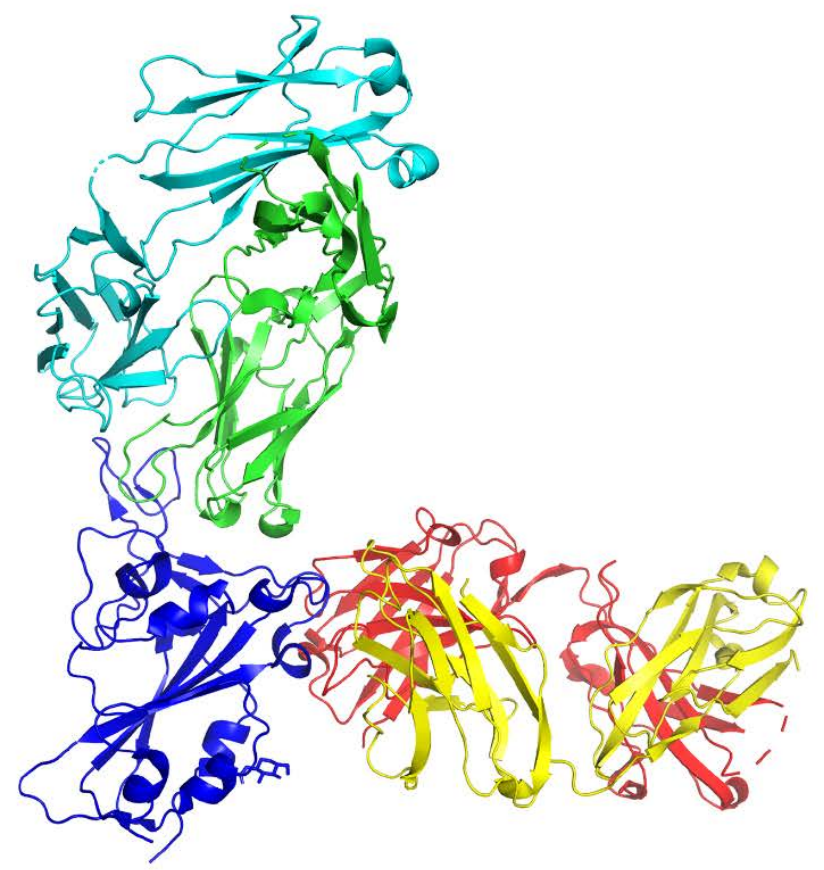

Fig. 4. Complex of REGN10933 and REGN10987 with the SARS-CoV-2 RBD. (A) $3.9 \AA$ cryoEM map of REGN10933 + RBD + REGN10987 complex, colored according to the chains in the refined model (B). RBD is colored dark blue, REGN10933 heavy and light chains are green and cyan, and REGN10987 heavy and light chains are yellow and red, respectively. 